\title{
歌iresalce
}

\section{A plataforma Kahoot!@ no ensino de histologia em um curso de medicina}

\author{
THE KAHOOT!® PLATFORM IN HISTOLOGY TEACHING IN A MEDICAL COURSE - \\ EXPERIENCE REPORT
}

Jardel de Almeida Monteiro', Maitê Assis Rodrigues², João Gabriel de Moura³ , Ana Paula da Silva Perez ${ }^{4}$

\author{
1 Graduando em Medicina. Universidade Federal de \\ Jataí (UFJ). \\ ORCID: https://orcid.org/0000-0001-8025-3603 \\ E-mail: jardelmonteiro@discente.ufg.br \\ 2 Graduando em Medicina. Universidade Federal de \\ Jataí (UFJ). \\ ORCID: https://orcid.org/0000-000 1-6996-7322 \\ E-mail: assis.maite@gmail.com \\ ${ }^{3}$ Graduando em Medicina. Universidade Federal de \\ Jataí (UFJ). \\ ORCID: https://orcid.org/0000-0001-7229-8537 \\ E-mail: naturezamoura@gmail.com \\ 4 Doutora em Biologia Celular e Estrutural. Universidade \\ Federal de Jataí (UFJ). \\ ORCID: https://orcid.org/0000-0002-2195-2452 \\ E-mail: paulabio_perez@ufg.br
}

Correspondência: Prédio de Medicina da Regional Jataí da UFG, Campus Jatobá (Cidade Universitária). Rodovia BR 364, Km 195, Setor Parque Industrial, $n^{\circ}$ 3800, Jataí-GO. CEP: 75801-615.

Copyright: Esta obra está licenciada com uma Licença Creative Commons Atribuição-NãoComercial 4.0 Internacional.

Conflito de interesses: os autores declaram que não há conflito de interesses.

\section{Como citar este artigo}

Monteiro JA; Rodrigues MA; De Moura JG; Perez APS. A plataforma Kahoot! ${ }^{\circledR}$ no ensino de histologia em um curso de medicina. Revista de Saúde Digital e Tecnologias Educacionais. [online], volume 5, n. 2. Editor responsável: Luiz Roberto de Oliveira. Fortaleza, mês e ano, p. 01$15 . \quad$ Disponível em: http://periodicos.ufc.br/resdite/index. Acesso em "dia/mês/ano".
Data de recebimento do artigo: 14/10/2019

Data de aprovação do artigo: 01/06/2020

Data de publicação: 20/07/2020

\section{Resumo}

Introdução: 0 avanço das Tecnologias de Informação e Comunicação (TICs) e as alterações nos perfis dos cursos da área da saúde exigem que a formação e o modo de mobilizar conhecimento se reinventem. Assim, não é novidade que os métodos tradicionais deem lugar às inovações das Metodologias Ativas de Aprendizagem e o uso de games no ambiente acadêmico. Objetivo: Relatar uma experiência de aprendizagem associada a jogos e tecnologias digitais no conteúdo de disciplinas básicas das áreas da saúde. Métodos: 0 estudo formata-se em relato de experiência sobre o uso do Kahoot! § ${ }^{\circledR}$ no contexto do ensino de histologia na educação médica. Resultados: 0 uso do jogo por meio das TICs, para que os estudantes mobilizassem os conteúdos, mostrou-se como uma ferramenta promissora no sentido de incentivar a motivação e o engajamento durante o processo de estudo. Conclusão: A experiência e o desempenho dos estudantes foram favoráveis, demonstrando que - Kahoot!® é uma tecnologia aplicável na educação superior em saúde no conteúdo das matérias básicas. Por fim, sugere-se que novos estudos sejam desenvolvidos para aprofundar as possibilidades de uso dessa tecnologia.

Palavras-Chave: Histologia. Aplicações da Informática Médica. Educação Médica. 


\section{Abstract}

Introduction: Advances in Information and Communication Technologies (ICTS) and changes in health course profiles change the way we mobilize knowledge, reinventing ways of learning. Unsurprisingly, traditional methods are replaced by innovations in Active Learning Methodologies and the use of games in the academic environment. Objective: Report a learning experience associated with games and digital technologies in the content of basic health disciplines. Methods: The study was formatted in relation to the experience of using Kahoot! $\mathbb{B}$ in the context of teaching histology in medical education. Results: The use of the game through
ICTs for students to mobilize the content proved to be a promising tool to encourage motivation and engagement during the study process. Conclusion: The experience and the students performance were favorable, showing that Kahoot! $\circledast$ is a possible and effective technology for higher education in health and the content of histology and other basic subjects. Finally, it is suggested that new studies to deepen the possibilities of using this technology.

Keywords: Histology. Medical Informatics Applications. Education, Medical.

\section{Introdução}

Com o progresso contínuo das Tecnologias de Informação e Comunicação (TICs), da engenharia eletrônica e das ciências médicas, faz-se mister que desponte um novo perfil de profissionais de saúde. Diante dessas circunstâncias, além das verdades construídas passarem a ser mais efêmeras, os pacientes estão cada vez mais informados por possuírem acesso aos conteúdos disponíveis na internet ${ }^{1}$.

Desse modo, a formação superior em saúde passa por profundas transformações que possibilitam desenvolver as habilidades necessárias ao egresso. Dentre essas aptidões, podem-se listar: prática baseada em evidências científicas, capacitação em novas tecnologias, competência comunicativa, capacidade de trabalho em equipe e autocrítica ${ }^{2}$.

Nessa perspectiva, surgem as Metodologias Ativas de Aprendizagem, por meio das quais o aprendizado deixa de ser passivo e o estudante passa a desempenhar um protagonismo, mobilizando e despertando competências necessárias ao novo perfil profissional. Tais abordagens são comprovadamente mais eficazes ${ }^{1}$.

Assim, a associação entre essas metodologias e o uso de TICs são capazes de promover uma formação médica mais atualizada, em que o profissional é capaz de se adaptar aos mutáveis contextos digitais e científicos. Esses métodos, apropriando-se dessas ferramentas tecnológicas, são promissores no processo de construção de um ambiente pedagógico sólido e estimulante (pois abaixo tem instigante novamente) ao estudante ${ }^{3}$.

A inserção de aparelhos eletrônicos, portanto, é oportuna e apropriada por imergir o aluno no contexto tecnológico e virtual, no âmbito acadêmico, por meio de uma ferramenta 
do seu cotidiano e permitir contato com ambientes que desenvolverão habilidades no manuseio de equipamentos da informática médica ${ }^{4,5}$.

Diante desse contexto, é indispensável trazer à tona meios eficazes, de baixo custo e inovadores, que, não só despertem o interesse, mas também sejam motivadores para a excelência acadêmica.

Desponta, nessa circunstância, o Kahoot!@, que se trata originalmente de uma plataforma norueguesa de jogos customizáveis voltados ao ensino. Essa ferramenta gratuita permite a confecção de quizzes que sejam respondidos em dispositivos eletrônicos remotos, como smartphones e tablets mediados pela internet. 0 jogo é disposto em perguntas com alternativas em formatos geométricos, associadas a cores, e conta com um temporizador em todas as perguntas. Ao final de cada resposta, o usuário tem acesso a um gráfico que mostra a alternativa correta e a quantidade de participantes que assinalaram cada uma das alternativas. Dessa forma, todo acerto é convertido em uma pontuação dependente do tempo despendido em cada resposta. Esses pontos acumulam-se até o final da sessão, gerando um ranking que fica disponível para o aplicador juntamente com as estatísticas de cada questão. Define-se também um pódio com os três primeiros colocados, que é público para todos os usuários.

Assim, a gamificação como estratégia pedagógica é possível graças ao uso do Kahoot!@ que, sendo uma ferramenta gratuita, é passível de ampla disseminação. Logo, a utilização desse jogo pode atuar sobre aspectos, como: tomadas de decisões, estabelecimento de estratégias, solução de problemas e potencialização da aprendizagem por meio do uso de linguagem visual e sonora que facilitam esse processo. O Kahoot!® ainda atribui uma nova perspectiva sobre a utilização da informática no meio médico e acadêmico, gerando novos vínculos entre o profissional de saúde e a tecnologia.

Sendo assim, o presente estudo tem por objetivo descrever a experiência do uso do Kahoot!@ em uma aula de Tecido Nervoso no conteúdo de Histologia, ministrada em uma universidade federal.

\section{Métodos}

Trata-se de um estudo descritivo, tipo relato de experiência, elaborado no contexto do Submódulo de Histologia, do módulo Estudo Morfofuncional do Corpo Humano Saudável I, ministrado no primeiro período do Curso de Medicina da Universidade Federal de Jataí. A atividade foi realizada no laboratório de Microscopia I do prédio da Medicina e tinha por 
objetivo o aprendizado do Tecido Nervoso, em termos de aspectos gerais, tipos celulares, caracterização do neurônio e descrição histológica do Sistema Nervoso Central (SNC) e Periférico (SNP).

Foi, portanto, explorada a Metodologia Ativa de Aprendizagem, por meio do Kahoot!@ (Noruega, <kahoot.com>), cuja proposta é o uso de jogos educativos em forma de quiz. No caso, o docente responsável pelo submódulo confeccionou dez questões envolvendo o objetivo da atividade, com quatro alternativas, sendo apenas uma correta.

Para a realização da atividade, a turma foi dividida em duplas, as quais utilizaram um celular com acesso à internet, por meio do qual as respostas do quiz seriam marcadas. Destaca-se que não era permitido o acesso aos materiais de consulta. Dessa forma, as perguntas do site foram apresentadas por meio de um projetor multimídia e as respostas foram marcadas pelas duplas, via celular. Cada pergunta poderia ser respondida em até 30 segundos e, ao final do tempo, os acertos eram convertidos em uma pontuação variável conforme o tempo empregado para marcar a alternativa correta. Essa pontuação se acumulou até o final do questionário, gerando um ranking (Tabela 1, ver Anexos).

A turma foi composta por 16 duplas participantes. Os questionamentos presentes e as respectivas alternativas são descritos a seguir (as alternativas destacadas são as respostas corretas):

Questão 1: Células pequenas e ativas na formação e manutenção da bainha de mielina do SNC:

a) Micróglias;

b) Oligodendrócitos;

c) Astrócitos;

d) Células de Schwann.

Questão 2: Cone axônico é:

a) Ponto de referência que distingue axônio de dendrito;

b) Importante região dos astrócitos;

c) Rico em organelas citoplasmáticas;

d) Nenhuma das alternativas.

Questão 3: Sobre o segmento inicial é INCORRETO afirmar:

a) Local onde o potencial de ação é gerado no axônio;

b) Região entre o ápice do cone axonal e o início da bainha;

c) Local identificado em todas as células da glia; 
d) Região encontrada nos neurônios.

Questão 4: A barreira hematoencefálica é formada por:

a) junções comunicantes entre as células do endotélio;

b) apenas pés vasculares dos astrócitos;

c) Zônulas de oclusão, lâmina basal e os pés vasculares;

d) Nenhuma das alternativas.

Questão 5: Camada delicada e vascularizada que repousa direto no encéfalo e na medula:

a) dura-máter;

b) aracnoide;

c) pia-máter;

d) células ependimárias;

Questão 6: Quando o SNC é danificado, as células gliais se mobilizam para formar a "cicatriz glial", sendo a:

a) fibrose;

b) inflamação;

c) gliose;

d) degeneração.

Questão 7: De acordo com o número de prolongamentos, os neurônios podem ser classificados em:

a) apenas unipolares;

b) multipolares, bipolares e pseudounipolares;

c) apenas bipolares;

d) motores e sensitivos.

Questão 8: Os corpos celulares dos neurônios dos gânglios são circundados por uma camada de pequenas células cuboides, denominadas:

a) Micróglias;

b) Células de Schwann;

c) Células satélites;

d) Células Ependimárias.

Questão 9: No SNP, (1) produzem uma camada rica em lipídios, a bainha de mielina, que circunda os axônios. (1) são:

a) Astrócitos;

b) Oligodendrócitos; 
c) Ependimárias;

d) Células de Schwann.

Questão 10: Identifique a célula cujos prolongamentos são pouco numerosos e grandes e o seu local de predomínio:

a) Astrócito fibroso, substância cinzenta;

b) Astrócito fibroso, substância branca;

c) Astrócito protoplasmático, substância cinzenta;

d) Astrócito protoplasmático, substância branca.

\section{Resultados}

Quando submetidos a testes e provas, os alunos são cobrados a demonstrarem um aproveitamento do conteúdo ministrado. No entanto, sabe-se que o rendimento é maior quando essas avaliações são utilizadas de maneira formativa e constituem o processo de ensino-aprendizagem, sendo elemento norteador dos processos desenvolvidos ${ }^{6}$.

Nessa lógica, a atividade foi realizada após uma exposição dialogada a respeito das características gerais e morfofuncionais, tipos celulares e descrição histológica do Tecido Nervoso, às 13h30, do terceiro dia do mês de outubro de 2019. Após a projeção do código de acesso ao quiz, ele foi inserido pelas duplas de alunos, via celular, no site do Kahoot!®e a atividade teve início. Vale destacar que, ao término, as duplas tiveram acesso instantâneo ao seu resultado e ao ranking de pontuação da turma, permitindo uma comparação geral de desempenho. A Figura 1 (anexos) é uma imagem demonstrativa de como funciona o quiz na plataforma. A Figura 2 (anexos) é a imagem que foi exibida pelo projetor multimídia no momento da questão 1.

A Figura 3 (anexos) é uma fotografia da atividade em realização, mostrando como os participantes viam as questões e com os celulares assinalaram a formas geométricas correspondentes às alternativas corretas. Os membros expostos na fotografia assinaram termo de autorização para esta publicação.

No decorrer da atividade, tornou-se evidente o engajamento e o foco dos alunos em busca de acertar o maior número de questões no menor tempo possível. Não houve dispersão da atenção e o desempenho da turma como um todo foi positivo, demonstrando a eficácia do método de aprendizagem aplicado. Tal resultado pode ser verificado na Tabela 1 (Anexos), cujo ranking das duplas foi elaborado de acordo com o tempo de resposta e a 
quantidade de acertos. É possível verificar, ainda, que a média geral da turma foi $81,25 \%$, a qual representa uma alta taxa de aproveitamento.

Além disso, a ferramenta Kahoot! $\circledast$ mostrou-se útil para identificar quais conteúdos foram mais assimilados pelos alunos, conforme a Tabela 2 (Anexos). As questões 5, 6 e 7 obtiveram $100 \%$ de acerto e a questão 3 obteve $43,75 \%$. Esse resultado permite ao professor avaliar quais os gargalos de aprendizagem e elaborar estratégias para sedimentar melhor os temas relevantes para a formação dos alunos.

\section{Discussão}

A associação entre dispositivos eletrônicos e o uso de games, como meios de ensino, despontam em Websites que oportunizam jogos on-line personalizados pelo elaborador da atividade, como é o caso do Kahoot! @ (B. Conforme exemplificado pelo relato de experiência, essa plataforma permite a confecção de jogos de perguntas e respostas rápidas de forma lúdica e interativa, associando as alternativas de respostas a cores diferentes e formatos geométricos (Figura 2), além de permitir o anexo de imagens nas perguntas, fator crucial para o ensino histológico.

Essa ferramenta possibilita efeitos favoráveis em desfechos pedagógicos na educação médica, resultando em um melhor desempenho nos exames posteriores e significativa motivação e engajamento por parte dos alunos. Isso porque, durante o ciclo básico, as matérias são apresentadas de forma muito mais expositiva e pouco diversificada, tornando o aluno alheio à aplicabilidade desse conteúdo. Contudo, ao utilizar novas metodologias, os professores e tutores têm a possibilidade de ampliar a visão dos alunos diante dessas matérias básicas ${ }^{7}$.

O uso do Kahoot! $\circledast$ na educação médica é encorajado não só por ser um promotor de engajamento e motivação, mas também por facilitar o direcionamento do estudo como uma importante ferramenta formativa. Os resultados demonstrados pela Tabela 1 , os quais sintetizam o desempenho visualizado pelas duplas imediatamente após o término do teste via ferramenta, são evidências da taxa de aprendizagem dos alunos. Dessa forma, os testes - aplicados pela plataforma - podem servir de direcionamento para aprofundar os estudos, auxiliar o aluno a avaliar seu desempenho como disparador de uma autorreflexão e propiciar aos instrutores aporte para aperfeiçoar suas práticas didáticas ${ }^{8}$.

Nesse contexto, sessões bem elaboradas de um quiz permitem que o aluno mobilize o conhecimento despertado em sala e o auxilie a criar um ambiente concentrado de estudo, 
além de serem motivadoras e construtivas. Logo, essas são atividades que corroboram para a excelência no ensino por facilitar o diálogo entre professor e alunos e entre os próprios colegas. Possibilitam, portanto, um padrão comparativo entre os discentes e um suporte colaborativo pelas atividades a serem desenvolvidas em equipes ${ }^{7 ; 8}$. Tal possibilidade de comparação pode ser evidenciada pela Tabela 2, indicando gaps de aprendizagem potencialmente úteis para subsidiar a prática pedagógica, em busca de nivelamento do conhecimento ministrado em sala de aula.

Nesse contexto, a competição saudável e o meio tecnológico, em geral, agregam valor pedagógico à prática ${ }^{9}$. Em nível universitário, esse game pode trazer o elemento desafio para os estudantes, que sempre procuram o melhor desempenho possível em menor tempo. No âmbito da experiência relatada, por exemplo, envolvendo conhecimento histológico, a performance geral da turma indicou uma média de $81,25 \%$, bastante superior ao mínimo exigido pela instituição de ensino (60\%). Tal situação é benéfica considerando que na prática médica é imprescindível ser resolutivo com excelência em um curto tempo. Assim, é possível verificar que diversas aptidões para a práxis médica podem ser inicialmente desenvolvidas durante as práticas de estudo nas matérias básicas ${ }^{7 ; 9}$.

A histologia, sendo parte deste escopo de conteúdos básicos, é uma dessas disciplinas em que o uso de games por meio do Kahoot! ${ }^{\circledR}$ pode ser profícuo e gerar excelentes resultados, promovendo melhores desempenhos em avaliações seguintes, em comparação às abordagens tradicionais ${ }^{8}$. Entre outros aspectos favoráveis ao uso do Kahoot!@ podem-se arrolar: a viabilização de um processo educativo reflexivo, por permitir que os alunos considerem seus desempenhos em determinado espaço de tempo diante dos colegas e do que era esperado; ressignificação dos tópicos básicos da graduação, uma vez que modifica a abordagem e permite ao aluno criar um significado próprio aos conteúdos ali exigidos; segurança do estudo realizado, pois sendo um elemento formativo, usado para mobilizar o conhecimento já adquirido, os jogos podem promover confiança diante daquilo que se conhece ${ }^{10}$.

A construção colaborativa do conhecimento é um fator preponderante no uso das metodologias ativas, especificamente, o Kahoot! $\circledast$. Isso porque ao trabalhar em grupos, é possível despertar competências necessárias ao trabalho em equipe, tais como habilidades comunicativas, capacidade de auxílio mútuo, raciocínio crítico e, por fim, maturidade social que os métodos ativos cooperativos despertam nos participantes ${ }^{11}$. 
Sabe-se também que os estudantes desses períodos possuem grandes expectativas a respeito da educação médica. Nesse contexto, as metodologias ativas e o incremento de tecnologias no ambiente de estudo mostraram-se positivas em corresponder e superar as expectativas dos estudantes frente às disciplinas básicas ${ }^{12}$.

Por fim, é importante considerar que o uso de tecnologias digitais durante as aulas contribui para o processo de ensino-aprendizagem e para o desenvolvimento primário de habilidades e competências necessárias para o trabalho em equipe. Além disso, possibilita que o egresso tenha pensamento crítico e busque reinventar, assim como em um processo de ensino-aprendizagem, a maneira de lidar com os novos perfis de pacientes ${ }^{2}$.

\section{Conclusão}

Abordagens pedagógicas diversificadas devem ser valorizadas e propagadas pelos ambientes educacionais. O uso do Kahoot! $\circledast$ no ensino da histologia mostrou-se promissor como ferramenta motivadora e eficaz em propiciar bons resultados.

Assim, a prática de ensino baseada em jogos, aliada ao uso de celulares, pode ser uma estratégia inovadora que permite aos jovens estudantes um engajamento e um direcionamento nos estudos que transformem o modo de desenvolver habilidades. Diante disso, é importante que os professores e tutores se atentem para novas e diferentes formas de mobilizar e agregar conhecimento, de modo a estimular diferentes perfis de ensino aos diferentes perfis de alunos. Isso porque é inegável o potencial que as tecnologias digitais móveis possuem e o domínio que os jovens estudantes têm delas.

Nessa perspectiva, o Kahoot!@ é uma estratégia possível e acessível para que o aprendizado da histologia seja sólido e envolvente para os estudantes. Diante do exposto, utilizar essa ferramenta é recomendável para a diversificação e o aprimoramento no processo de agregar conhecimento aos discentes de forma interativa, colaborativa e provocativa. Ainda assim, são necessários estudos aprofundados sobre o tema, quantitativos e/ou qualitativos, que evidenciem a eficácia dessa ferramenta como agente motivador e promotor de melhores resultados em longo prazo. Ademais, considerando que o Kahoot! é amplamente utilizado, essa experiência poderia ser replicada para outros campos de ensino, cujos resultados poderiam ser comparados aos obtidos nesse relato. 


\section{Referências}

1. Farias PAM; Martin ALAR; Cristo CS. Aprendizagem ativa na educação em saúde: percurso histórico e aplicações. Revista brasileira de educação médica, v. 39, n. 1, p. 143150, 2015. Disponível em: http://www.scielo.br/scielo.php?pid=S010055022015000100143\&script=sci_abstract\&tlng=pt

2. Berbel NAN. As metodologias ativas e a promoção da autonomia de estudantes. Semina: Ciências Sociais e Humanas, [s.I.], v. 32, n. 1, p.25-40, 27 mar. 2011. Universidade Estadual de Londrina. Disponível em:

http://www.uel.br/revistas/uel/index.php/seminasoc/article/view/10326/0

3. De Carvalho CV. APRENDIZAGEM BASEADA EM JOGOS GAME-BASED LEARNING. In: II World Congress on Systems Engineering and Information Technology. 2015. p. 176-181. Disponível em:https://copec.eu/congresses/wcseit2015/proc/works/40.pdf

4. Geladze D. Using the Internet and Computer Technologies in Learning/Teaching Process. Journal of Education and Practice, v. 6, n. 2, p. 67-69, 2015. Disponível em: https://files.eric.ed.gov/fulltext/EJ1083835.pdf

5. Amem BMV; Nunes LC. Tecnologias de Informação e Comunicação: contribuições para o processo interdisciplinar no ensino superior. Revista brasileira de educação médica. Rio de Janeiro, v.30, n.3, p. 171-180, 2006. Disponível em:

http://www.scielo.br/scielo.php?pid=s0100$55022006000300008 \&$ script $=$ sci_abstract\&tlng=pt

6. Chizzotti A. Políticas públicas: direito de aprender e avaliação formativa. Práxis Educativa (Brasil) [Internet]. 2016;11(3):561-576. Disponível em:http://portal.amelica.org/ameli/jatsRepo/89446960002

7. Jamil Z; Fatima SS; Saeed AA. Preclinical medical students' perspective on technology enhanced assessment for learning. JPMA, v. 68, n. 898, 2018. Available from: https://www.ncbi.nlm.nih.gov/pubmed/30325908.

8. Ismail M Al-A, et al. Using Kahoot! as a formative assessment tool in medical education: a phenomenological study. BMC medical education, v. 19, n. 1, p. 230, 2019. Available from: https://bmcmededuc.biomedcentral.com/articles/10.1186/s12909-019-1658-z

9. Felszeghy $S$, et al. Using online game-based platforms to improve student performance and engagement in histology teaching. BMC medical education, v. 19, n. 1, p. 273, 2019. Available from: https://bmcmededuc.biomedcentral.com/articles/10.1186/s12909-0191701-0

10. Castro MJ, et al. Impact of educational games on academic outcomes of students in the Degree in Nursing. PloS one, v. 14, n. 7, p. e0220388, 2019. Available from: https://www.ncbi.nlm.nih.gov/pmc/articles/PMC6663014/ 11. Conceição CV; Moraes MAA. Aprendizagem Cooperativa e a Formação do Médico Inserido em Metodologias Ativas: um Olhar de Estudantes e Docentes. Revista brasileira de educação médica, Brasília, v. 42, n. 4, p. 115-122, 2018. Disponível em: http://www.scielo.br/scielo.php?script=sci_abstract\&pid=S010055022018000400115\&lng=en\&nrm=iso\&tlng=pt

12. Meireles MAC; Fernandes CCP; Silva LSE. Novas Diretrizes Curriculares Nacionais e a Formação Médica: Expectativas dos Discentes do Primeiro Ano do Curso de Medicina de uma Instituição de Ensino Superior. Revista brasileira de educação médica, Brasília, v. 43, n. 2, p. 67-78, 2019. Disponível em:

http://www.scielo.br/scielo.php?script=sci_abstract\&pid=S0100-

55022019000200067\&lng=pt\&nrm=iso 
13. Munguba MC, et al. Jogos eletrônicos: apreensão de estratégias de aprendizagem. Revista Brasileira em Promoção da Saúde, v. 16, n. 1, p. 39-48, 2012. 


\section{Anexos}

\section{Lista de figuras}

Figura 1: Imagem ilustrativa do Kahoot!®

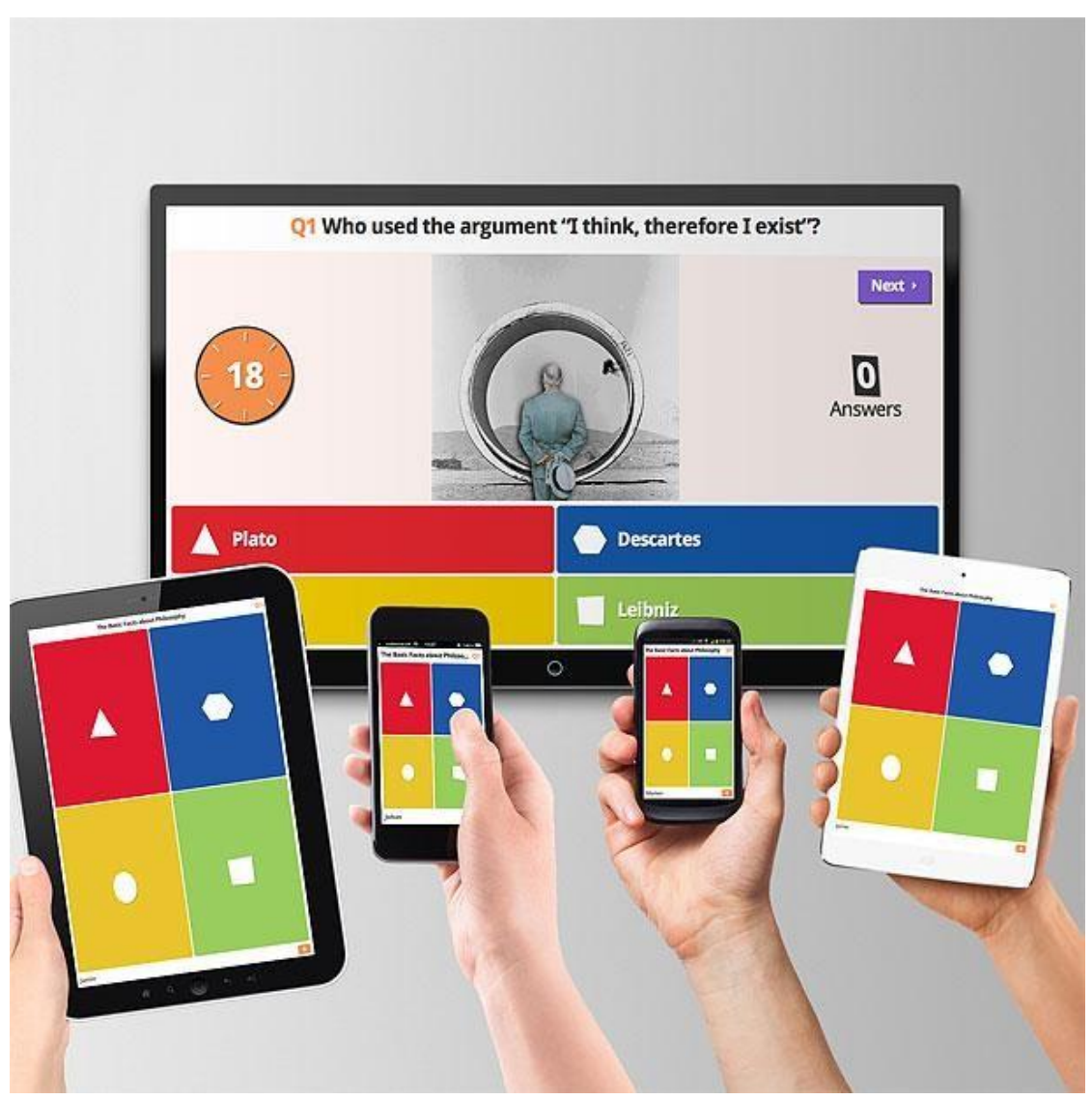

Fonte: https://play.kahoot.it/

Figura 2: Imagem ilustrativa do Kahoot! ${ }^{\circledR}$

Células pequenas e ativas na formação e manutenção da bainha de mielina do SNC.

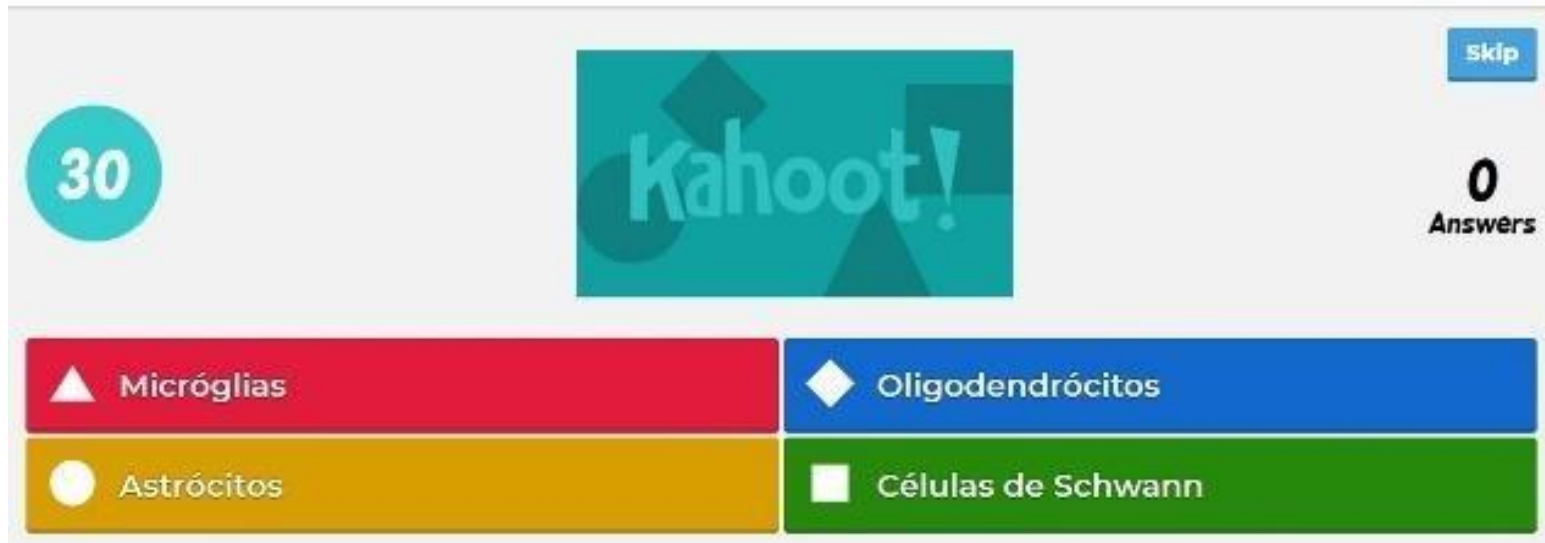

Fonte: dos Autores. 
Figura 3: fotografia da atividade em realização.

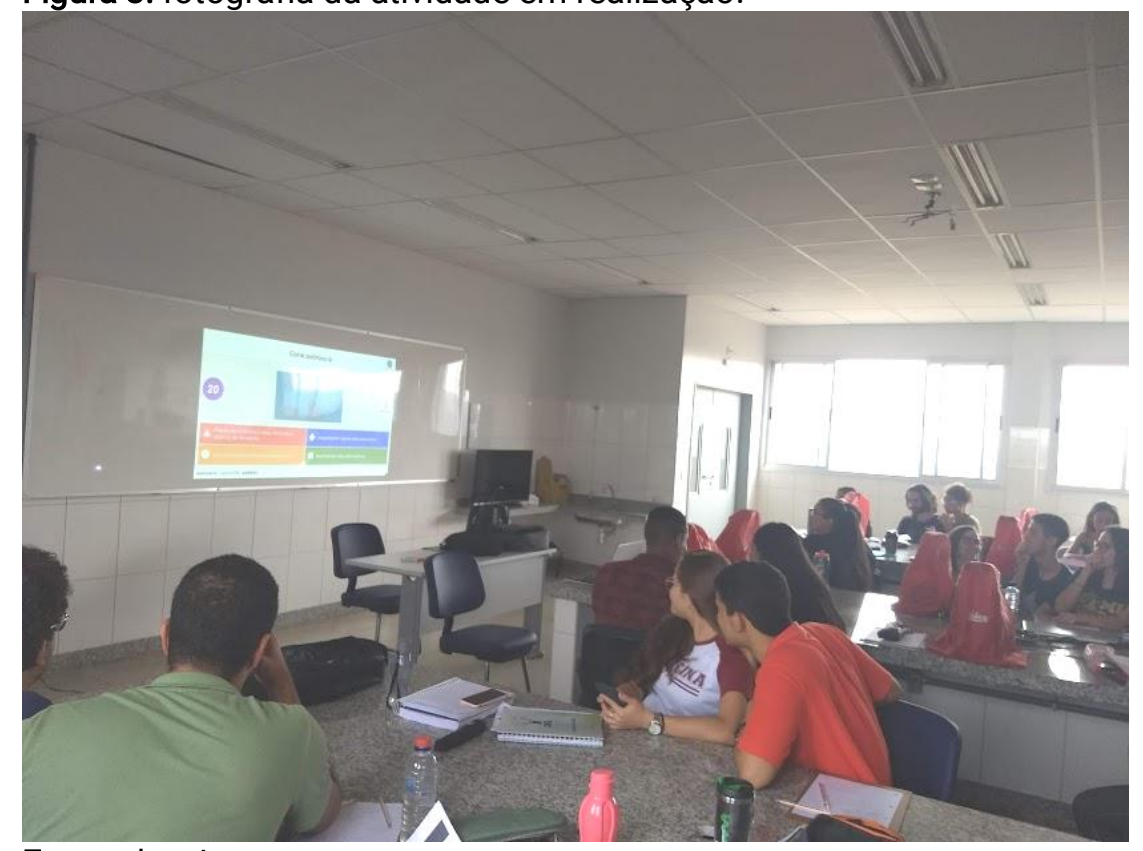

Fonte: dos Autores.

Tabela 1. Ranking das duplas jogadoras ${ }^{a}$

\begin{tabular}{|c|c|c|c|c|}
\hline Classificação das duplas & Pontuação final & Acertos & Erros & Percentual de acerto (\%) \\
\hline 1 & 12013 & 10 & 0 & 100 \\
\hline 2 & 10896 & 9 & 1 & 90 \\
\hline 3 & 10876 & 10 & 0 & 100 \\
\hline 4 & 10690 & 9 & 1 & 90 \\
\hline 5 & 9825 & 8 & 2 & 80 \\
\hline 6 & 9540 & 8 & 2 & 80 \\
\hline 7 & 9430 & 9 & 1 & 90 \\
\hline 8 & 9383 & 9 & 1 & 90 \\
\hline 9 & 9049 & 8 & 2 & 80 \\
\hline 10 & 9034 & 7 & 3 & 70 \\
\hline
\end{tabular}




\begin{tabular}{lllll}
11 & 8999 & 8 & 2 & 80 \\
12 & 8379 & 7 & 3 & 70 \\
13 & 7979 & 8 & 2 & 80 \\
14 & 7732 & 7 & 3 & 70 \\
15 & 7666 & 7 & 3 & 70 \\
16 & 6415 & 6 & 4 & 60 \\
\hline Média geral & 9244,125 & 8,125 & 1,875 & 81,25
\end{tabular}

aRealizada com base nos dados fornecidos pela plataforma, levando em consideração tempo de resposta e número de acertos. Fonte: aos autores.

Tabela 2. Análise das respostas ${ }^{a}$

\begin{tabular}{|c|c|c|c|c|c|c|}
\hline \multirow[t]{2}{*}{ Questão } & \multirow{2}{*}{$\begin{array}{l}\text { Percentual de } \\
\text { duplas corretas } \\
\text { (\%) }\end{array}$} & \multirow{2}{*}{$\begin{array}{l}\text { Alternativa } \\
\text { correta }\end{array}$} & \multicolumn{4}{|c|}{ Quantidade de respostas por alternativa } \\
\hline & & & $A$ & B & $\mathrm{C}$ & $\mathrm{D}$ \\
\hline 1 & 81,25 & B & 1 & 13 & 0 & 2 \\
\hline 2 & 68,75 & $A$ & 11 & 1 & 3 & 1 \\
\hline 3 & 43,75 & C & 6 & 1 & 7 & 0 \\
\hline 4 & 75,00 & C & 1 & 2 & 12 & 0 \\
\hline 5 & 100,00 & C & 0 & 0 & 16 & 0 \\
\hline 6 & 100,00 & C & 0 & 0 & 16 & 0 \\
\hline 7 & 100,00 & B & 0 & 16 & 0 & 0 \\
\hline 8 & 93,75 & C & 0 & 0 & 15 & 1 \\
\hline 9 & 93,75 & D & 0 & 1 & 0 & 15 \\
\hline
\end{tabular}


10

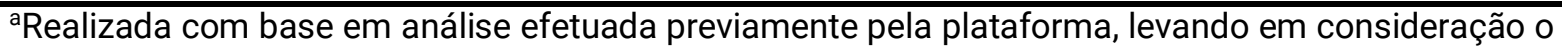
percentual de duplas corretas e a quantidade de respostas por alternativa. Fonte: Elaborada pelos autores. 